\section{A) Check for updates}

Cite this: Photochem. Photobiol. Sci., 2020, 19, 1160

\title{
Remodelling of fibrillin-rich microfibrils by solar-simulated radiation: impact of skin ethnicity
}

\author{
Abigail K. Langton, (D) *a,b Mark Hann, ${ }^{c}$ Patrick Costello, ${ }^{a}$ Poonam Halai, ${ }^{a}$ \\ Christopher E. M. Griffiths, ${ }^{a, b}$ Michael J. Sherratt ${ }^{d}$ and Rachel E. B. Watson (D) ${ }^{a, b}$
}

\begin{abstract}
Fibrillin-rich microfibrils (FRMs) constitute integral components of the dermal elastic fibre network with a distinctive ultrastructural 'beads-on-a-string' appearance that can be visualised using atomic force microscopy and characterised by measurement of their length and inter-bead periodicity. Their deposition within the dermis in photoprotected skin appears to be contingent on skin ethnicity, and influences the ultrastructure of papillary - but not reticular - dermal FRMs. Truncation and depletion of FRMs at the dermal-epidermal junction of skin occurs early in photoageing in people with lightly pigmented skin; a process of accelerated skin ageing that arises due to chronic sun exposure. Accumulation of ultraviolet radiation (UVR)-induced damage, either by the action of enzymes, oxidation or direct photon absorption, results in FRM remodelling and changes to ultrastructure. In the current study, the direct effect of UVR exposure on FRM ultrastructure was assayed by isolating FRMs from the papillary and reticular dermis of photoprotected buttock skin of individuals of either black African or white Northern European ancestry and exposing them to solar-simulated radiation (SSR). Exposure to SSR resulted in significant reduction in inter-bead periodicity for reticular dermis-derived FRMs across both cohorts. In contrast, papillary dermal FRMs exhibited significantly increased inter-bead periodicity, with the magnitude of damage greater for African FRMs, as compared to Northern European FRMs. Our data suggest that FRMs of the dermis should be considered as two distinct populations that differentially accrue damage in response to SSR. Furthermore, papillary dermal FRMs derived from black African subjects show greater change following UVR challenge, when extracted from skin. Future studies should focus on understanding the consequences of UVR exposure in vivo, regardless of skin ethnicity, on the molecular composition of FRMs and how this UVR-induced remodelling may affect the role FRMs play in skin homeostasis.
\end{abstract}

Received 15th May 2020, Accepted 27 th June 2020 DOI: $10.1039 / \mathrm{d} 0 \mathrm{pp} 00188 \mathrm{k}$ rsc.li/pps and recoil many times over the lifetime of an individual. ${ }^{1,2}$ During early development, the genesis of elastic fibres involves the deposition of tropoelastin (the soluble precursor of mature elastin) on a pre-formed template of $\mathrm{FRMs}^{3}$ and, regardless of tissue type or species, these have a characteristic 'beads-on-astring' ultrastructural appearance with an average inter-bead distance (or periodicity) of $56 \mathrm{~nm} ;{ }^{4}$ however, FRM and the composition of their accessory proteins is tissue-specific. ${ }^{5}$

The elastic fibre network forms a distinctive, highly ordered arrangement within the dermal ECM: at the dermal-epidermal junction (DEJ), superficial oxytalan fibres consist of cascades of discrete FRM bundles. These 'oxytalan' fibres coalesce with a fine network of elastin and fibrillin-rich 'elaunin' fibres within the papillary dermis, whilst in the reticular dermis, mature elastic fibres run in parallel to the DEJ. ${ }^{6,7}$ In young, healthy photoprotected skin we have previously identified that FRMs are differentially deposited in the papillary dermis of individuals from diverse ethnic backgrounds; with black African skin containing significantly more FRMs than both white Northern European and Far East Asian skin types. ${ }^{8}$ 
Furthermore, it has recently been demonstrated that skin ethnicity also influences the ultrastructure of FRMs. ${ }^{9}$ At birth, FRM ultrastructure is invariant; however, in adults from diverse ethnic backgrounds, there is a significant difference in ultrastructure for papillary dermal FRMs. In contrast, reticular dermal FRMs are invariant between individuals with diverse skin ethnicities, at least in regards to their periodicity. ${ }^{9}$

Depletion of FRMs at the DEJ is a characteristic feature of early photoageing in white Northern European individuals and arises early in response to chronic sun exposure, ${ }^{10}$ and can lead to skin laxity ${ }^{11}$ and accelerated ageing. ${ }^{10}$ Exposure of lightly-pigmented skin to ultraviolet radiation (UVR) induces a complex cascade of detrimental ECM remodelling events, such as infiltration of immune cells, ${ }^{12}$ photo-oxidation ${ }^{13}$ and release and activation of sequestered matrix metalloproteinases (MMPs). ${ }^{14}$ However, due to the protective nature of melanin, ${ }^{15,16}$ individuals with highly-pigmented skin do not succumb to equivalent levels of ECM remodelling until much later in life. ${ }^{17}$ Here, we assay the direct effect of solar simulated radiation (SSR) exposure on FRM ultrastructure by irradiating FRMs following their extraction from black African and white Northern European skin. Hence, in this experimental system, FRMs are not afforded protection from melanin, and the usual remodelling events that would occur in a physiological setting are negated.

\section{Methods}

\section{Skin biopsy procurement}

Young healthy black African ("African"; $n=5$, mean age \pm SD: 21.2 years $\pm 1.9 ; 2 \mathrm{M}$, 3F; Fitzpatrick skin type (FST) VI) and white Northern European ("European"; $n=5,22.4$ years \pm 2.3 ; $2 \mathrm{M}, 3 \mathrm{~F}$; FST I-II) volunteers were recruited to the study. Local ethical approval was obtained from the North West Research Ethics Committee (ref. 09/H1006/23) and the University of Manchester Research Ethics Committee (ref. 14161). Basic demographic information was collected and participants were asked to self-declare their ethnicity. Six (6) $\mathrm{mm}$ diameter punch biopsies were obtained from photoprotected buttock under $1 \%$ lignocaine local anesthesia. At the time of procurement, biopsies were bisected, snap frozen in liquid nitrogen and stored at $-80 \mathrm{C}$. All studies were conducted in accordance with the Declaration of Helsinki Principles with written, informed consent.

\section{Fibrillin microfibril isolation}

Assemblies of fibrillin-rich microfibrils were isolated from the papillary dermis of adult buttock skin by cryosectioning bisected $6 \mathrm{~mm}$ skin biopsies en face to a depth of $400 \mu \mathrm{m}$. Next, $100 \mu \mathrm{m}$ was cryosectioned from the skin biopsy and discarded; FRM were then isolated from the remaining reticular dermal fraction of the skin biopsy (Fig. 2a). Papillary and reticular dermal fractions were digested overnight in $0.5 \mathrm{mg} \mathrm{mL}^{-1}$ bacterial collagenase type IA (suspended in $0.4 \mathrm{M} \mathrm{NaCl}, 0.05 \mathrm{M}$ Tris-HCl, $0.01 \mathrm{M} \mathrm{CaCl}_{2}$ at $\mathrm{pH}$ 7.4, and supplemented with pro- tease inhibitors: $2 \mathrm{mM}$ phenylmethanesulfonyl fluoride and $5 \mathrm{mM} \mathrm{N}$-ethylmaleimide) ${ }^{18}$ Extracts were subsequently purified by low pressure size-exclusion chromatography on an ÄKTA prime plus system coupled to a Sepharose CL-2B column (GE Healthcare, Little Chalfont, UK) which was equilibrated in high salt buffer (0.4 M NaCl, $0.05 \mathrm{M}$ Tris- $\mathrm{HCl}$ at $\mathrm{pH}$ 7.4). FRMs were eluted from the column in the excluded volume $\left(V_{0}\right)$ peak.

Suspensions containing FRM were irradiated at an environmentally relevant dose of $15.4 \mathrm{~J} \mathrm{~cm}^{-2}$ in $2 \times 10 \mathrm{~mm}$ (height $\times$ diameter) polyethylene lids (total volume $250 \mu \mathrm{L}$ ) using a Solar Simulator (Applied Photophysics, Cambridge, UK; UVA; 95.0\%; UVB: $5.0 \%$ ) consisting of a xenon arc lamp filtered with a WG320 filter (Schott, Stafford, UK). All suspensions were incubated at room temperature for the duration of the SSR exposure. Spectral outputs of the solar simulator were measured using a double grating spectroradiometer (Bentham Instruments Ltd, Reading, UK) calibrated to National Physical Laboratory (Teddington, UK) standards. Routine irradiance measurements were made using a UVX radiometer and UVX-36 detector (UVR Products; Upland, CA, USA) calibrated against the spectroradiometer measurements for SSR (irradiance $=$ $54.3 \mathrm{~mW} \mathrm{~cm}^{-2}$ ) spectral output.

\section{Atomic force microscopy and data processing}

The ultrastructure of extracted FRMs were characterised by atomic force microscopy (AFM). Using the Multimode 8 AFM (Bruker AFM Probes, Camarillo, California USA) fitted with ScanAsyst-Air cantilevers, randomly selected $10 \times 10 \mu \mathrm{m}$ locations were scanned at a rate of $1.97 \mathrm{~Hz}$. The morphologic metrics assessed were the number of beads per FRM and inter-bead periodicity. Periodicity was determined by measuring the distance between individual beads $(n=1000)$ using WSxM scanning probe microscopy software and by routines written in Microsoft Visual Basic 6.0. Inter-bead periodicity is a widely used, reliable and quantitative marker for analysis of FRM ultrastructure. ${ }^{5,9,19-21}$

\section{Statistical analyses}

Regression modelling was conducted using Stata V15 software (StataCorp. 2017. Stata Statistical Software: Release 15. College Station, TX: StataCorp LLC) in order to determine whether differences in periodicity existed between the two groups, controlling for participant age and gender. As the data were hierarchical - inter-bead periodicity is 'clustered' within FRM which, themselves are clustered with participants - we initially fitted a multi-level mixed-effects linear regression model, which takes account of both the potential correlation between periodicity measurements within the same FRM and the potential correlation of FRMs within the same participant (although the latter is likely to be smaller). As the sample of periodicity measurements has some positive skew and is highly kurtotic, a non-parametric bootstrapped standard error was also derived, using 200 replications and a random initialvalue 'seed'. Having run these models, it was apparent that there was some mis-specification, as there were inconsistencies 
Table 1 Number of beads per fibrillin-rich microfibril before and after a single dose of $15.4 \mathrm{~J} \mathrm{~cm}^{-2}$ SSR irradiation

\begin{tabular}{lcll}
\hline & \multicolumn{2}{l}{ Number of beads per FRM [median (IQR)] } & \\
\cline { 2 - 4 } & Unirradiated & Irradiated & Significance \\
\hline African papillary dermis & $8.5(8.0,11.5)$ & $10.0(7.0,10.3)$ & $18.0(17.5,20.5)$ \\
African reticular dermis & $17.0(15.0,23.5)$ & $10.0(8.5,12.3)$ & $P>0.999$ \\
European papillary dermis & $9.0(8.5,11.8)$ & $21.0(18.0,26.0)$ & $P=0.579$ \\
European reticular dermis & $18.5(16.8,23.3)$ & & $P=0.643$ \\
& &
\end{tabular}

Table 2 Inter-bead periodicity before and after a single dose of $15.4 \mathrm{~J} \mathrm{~cm}^{-2} \mathrm{SSR}$ irradiation

\begin{tabular}{llcl}
\hline & \multicolumn{3}{l}{ Inter-bead periodicity [mean (std deviation) in nm] } \\
\cline { 2 - 4 } & Unirradiated & Irradiated & Significance \\
\hline African papillary dermis & $55.13(10.74)$ & $58.82(15.18)$ & $P<0.001$ \\
African reticular dermis & $56.73(12.37)$ & $58.63(11.20)$ & $P<0.001$ \\
European papillary dermis & $56.74(11.92)$ & $52.51(10.81)$ & $P<0.001$ \\
European reticular dermis & $56.59(11.32)$ & $P<0.001$
\end{tabular}

in the estimated standard errors. We therefore decided to take account of the likely correlation between any randomly chosen periodicity measurement and its preceding one in the 'chain' (known as auto-regression). We therefore fitted separate population-averaged linear regression models, with auto-regressive order-1 correlations (AR1) and robust standard errors (as bootstrapping is not available for this type of model), to the original data, to logarithmic-transformed data and to square roottransformed data.

\section{Results}

Buttock skin biopsies were dissected into papillary and reticular dermal components and FRMs were extracted prior to irradiation with a single dose of SSR $\left(15.4 \mathrm{~J} \mathrm{~cm}^{-2}\right)$, to allow characterisation of ultrastructural parameters (bead number per FRM and inter-bead periodicity; Fig. 1). For both papillary and reticular dermal FRMs, SSR exposure did not induce significant changes to the number of beads per microfibril in either cohort. However, reticular dermal FRMs consistently had a greater number of beads per FRM as compared to those extracted from the papillary dermis (Table 1).

Next, the impact of SSR exposure on inter-bead periodicity was assessed for both papillary and reticular dermal FRMs. For FRMs extracted from the papillary dermis, SSR caused a significant increase in inter-bead periodicity compared to unirradiated controls for both cohorts (Table 2). Using statistical modelling and regardless of whether the original-, logarith-
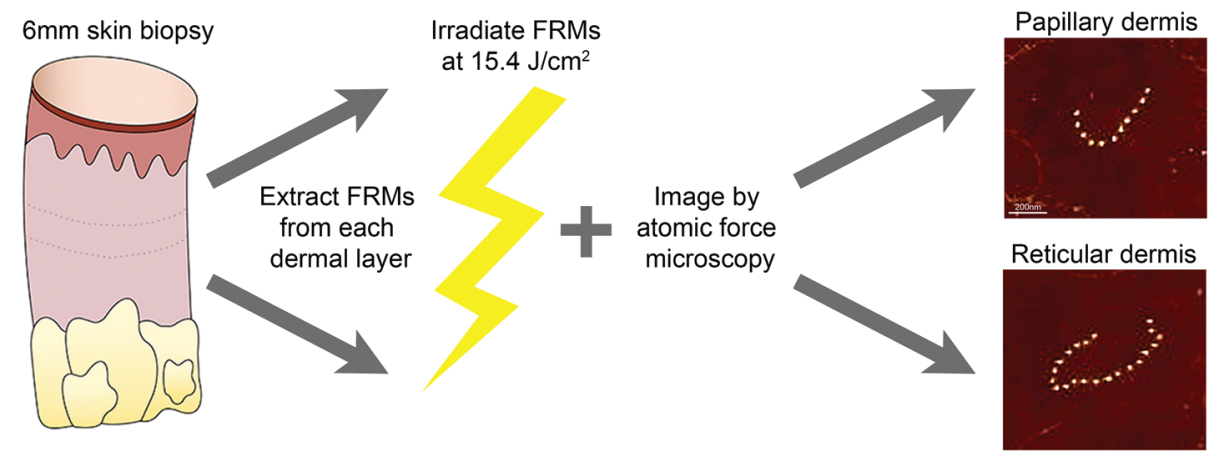

Fig. 1 Schematic methodology for the extraction and ex vivo irradiation of fibrillin-rich microfibrils (FRMs). Experimental isolation of FRMs from the papillary dermis of adult buttock skin was performed by cryosectioning bisected $6 \mathrm{~mm}$ skin biopsies en face to a depth of $400 \mu \mathrm{m}$. The next $100 \mu \mathrm{m}$ was cryosectioned from the skin biopsy and discarded. Reticular dermal FRMs were then isolated from the remaining skin biopsy. Extracted FRM were irradiated with a single dose of SSR $\left(15.4 \mathrm{~J} \mathrm{~cm}^{-2}\right)$ and imaged using atomic force microscopy. Representative atomic force microscopy images of FRM isolated from human papillary and reticular dermis reveal the "beads-on-a-string" morphology. Measurement of the inter-bead region provides a measure of FRM ultrastructure termed periodicity. Scale bar $=200 \mathrm{~nm}$. 
a Papillary Dermis
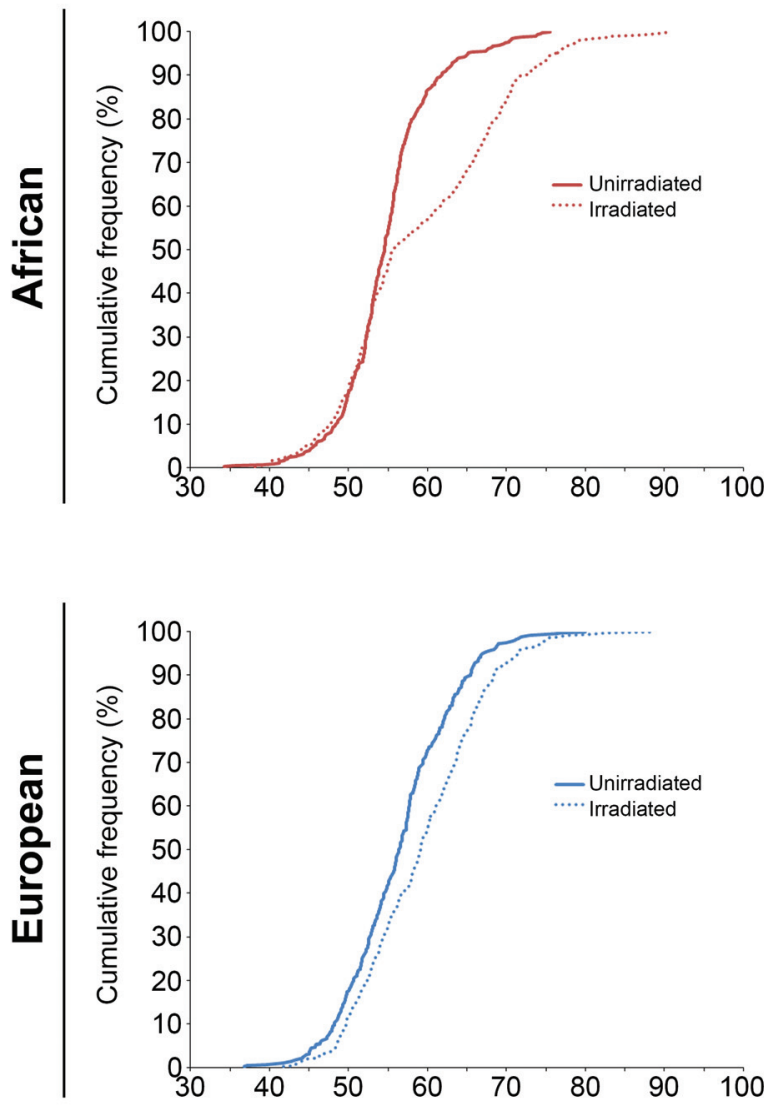

b

\section{Reticular Dermis}
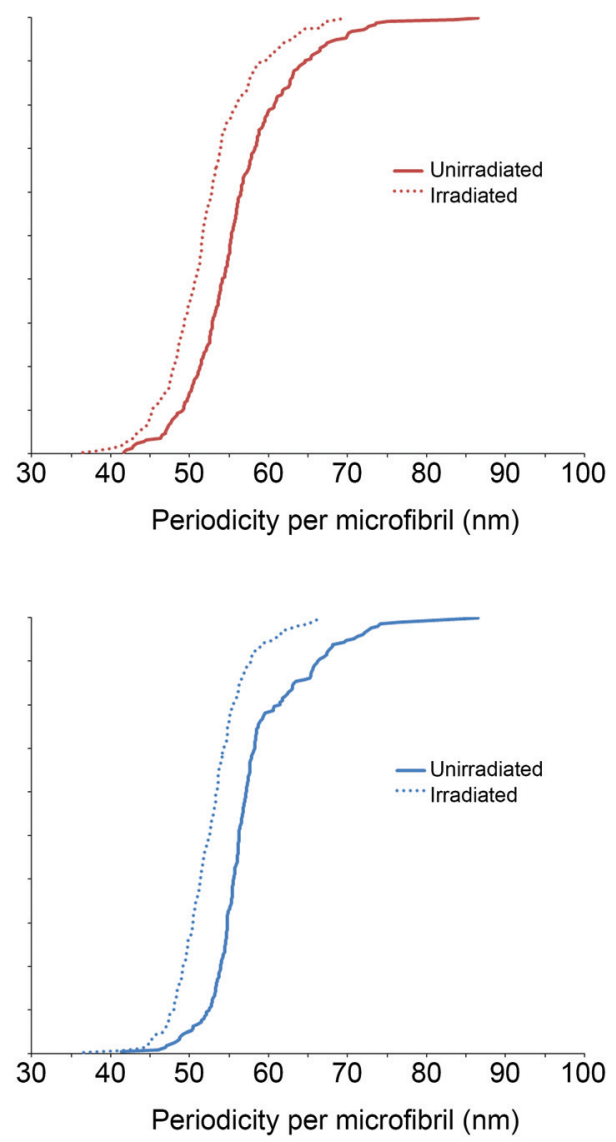

Fig. 2 Solar-simulated radiation induces differential remodelling of FRMs in the dermis of ethnically diverse skin. For FRMs extracted from the papillary dermis, SSR irradiation caused a significant increase to inter-bead periodicity compared to unirradiated controls for both African and European cohorts. However, the cumulative distributions revealed differences in the magnitude of each cohort's response to SSR, with the African response greater than that observed for the European cohort (a). In contrast, for FRMs extracted from the reticular dermis, SSR exposure caused a significant decrease to inter-bead periodicity as compared to unirradiated controls across both cohorts (b).

mic- or square root-transformed data were used, periodicity was significantly larger in irradiated FRMs. For ease of interpretation, we display here the original data. Controlling for age and gender, the regression parameters represent an adjusted mean difference in periodicity for African control FRM as compared to those SSR-exposed of $-3.71 \mathrm{~nm}$ [95\% C.I. $(-4.58 \mathrm{~nm},-2.83 \mathrm{~nm}) ; P<0.001]$ and for Northern European control as compared to those SSR-exposed of $-2.69 \mathrm{~nm}[95 \%$ C.I. $(-3.61 \mathrm{~nm},-1.78 \mathrm{~nm}) ; P<0.001]$. Furthermore, examination of the cumulative distributions revealed differences in each cohort's response to SSR, with the magnitude of the African response greater than that observed for the European cohort (Fig. 2).

In contrast, for FRMs extracted from the reticular dermis, SSR exposure caused a significant reduction in inter-bead periodicity as compared to unirradiated controls across both cohorts. Using statistical modelling and controlling for age and gender, the difference in reticular dermal FRM periodicity for African control as compared to SSR-exposed was $4.22 \mathrm{~nm}$ [95\% C.I. $(3.17 \mathrm{~nm}, 5.28 \mathrm{~nm}) ; P<0.001]$ and for Northern European control as compared to SSR-exposed was $3.96 \mathrm{~nm}$ [95\% C.I. $(2.76 \mathrm{~nm}$, $5.16 \mathrm{~nm}) ; P<0.001]$. Examination of the cumulative distributions revealed similarities in each cohort's response to SSR, with the magnitude of the African response similar to that observed for the Northern European cohort (Fig. 2).

Taken together, these findings show that experimental SSR does not cause significant changes to FRM bead number; however, it does induce changes to inter-bead periodicity. Furthermore, the direction of this difference appears to be dependent on the dermal compartment from which the FRMs are extracted $(P<0.001)$ but not from the ethnic group from which they are derived $(P=0.205)$. 


\section{Discussion}

In this study, we established that FRMs isolated from the papillary and reticular dermis of photoprotected buttock skin accrue significant changes to their inter-bead periodicity upon exposure to SSR. Papillary dermal FRMs when exposed to SSR exhibit significantly increased inter-bead periodicity whereas reticular dermal FRMs have significantly decreased inter-bead periodicity. Furthermore, the magnitude of this change for papillary dermal FRMs is significantly greater for the African cohort as compared to the European cohort.

The use of periodicity as a characteristic measure of ultrastructure is widely used as a reliable quantitative marker for the analysis of FRMs. ${ }^{19-21}$ Inter-bead periodicity of isolated, control FRMs is often reported as approximately $56 \mathrm{~nm} .^{4,22}$ However, we have previously demonstrated that FRMs exhibit a wide distribution of inter-bead periodicities, and that the reporting of a single value may underestimate the differences between samples. ${ }^{9}$ Therefore, this study employs microfibrilby-microfibril analysis of each FRM to provide detailed observations of the distribution of inter-bead periodicities.

Our experimental protocol was designed to assay the direct effect of SSR-exposure on FRM ultrastructure. This was achieved by irradiating the FRMs following their extraction from the skin and hence the usual events that would occur in a physiological setting - such as infiltration of immune cells into the skin, ${ }^{12}$ photo-oxidation ${ }^{13}$ or induction and release of MMPs $^{14}$ - were absent. The finding that bead number per FRM was unaffected by SSR exposure suggests that when fragmentation and truncation of FRM in photoexposed skin occurs, it is likely via a cell-mediated process driven by the UVR-induced expression and/or activation of ECM proteases, such as MMPs, ${ }^{23-25}$ and/or the liberation of reactive oxygen species (ROS) ${ }^{26}$ from the dermal ECM. It has recently been shown in vitro that structural changes to extracted FRM can be induced by UVB-irradiation and that these changes are largely ROS-driven. ${ }^{27}$ Therefore, we cannot entirely dismiss the role that photo-oxidation may play in our experimental system.

Further in vivo experiments (involving in vivo SSR exposure of human skin) may allow assessment of the protection that melanin affords individuals cross a range of ethnicities on FRM structure, similar to that shown using DNA damage as a measurable endpoint. ${ }^{15}$ However, this methodology has technical limitations which are challenging; even mild exposure to UVR in lightly-pigmented skin causes bundles of FRM within the papillary dermis to be severely truncated and for some individuals almost entirely depleted, ${ }^{10,11}$ making their extraction technically difficult.

Our choice of SSR dose $\left(15.4 \mathrm{~J} \mathrm{~cm}^{-2}\right)$ was selected as it was expected that this would give rise to reproducible alterations in FRM ultrastructure, consistent with the work of others and be a physiologically attainable dose. ${ }^{19,20}$ Following irradiation, alterations to FRM ultrastructure are often model- and irradiation source-specific; however, regardless of the microfibril and irradiation source, exposure to UVR reproducibly induces measurable changes in microfibril appearance. ${ }^{19,20}$ In the current study, it was somewhat unexpected that for human skin, FRMs isolated from different dermal layers show marked differences in the direction of change for inter-bead periodicity following SSR exposure, evidencing stochastic damage, and highlighting the importance of the model system used.

We have previously demonstrated that FRMs extracted from adult photoprotected buttock skin show heterogeneity; reticular dermal FRMs are long assemblies that have a consistent periodicity whereas papillary dermal FRMs have approximately half the bead number of their reticular counterparts and have marked differences in their periodicity between ethnic cohorts. ${ }^{9}$ Furthermore, we hypothesised that FRMs of the reticular dermis are likely derived from a fibroblast population, ${ }^{28}$ whereas papillary dermis-derived FRMs may be a product of both basal epidermal keratinocytes and papillary dermal fibroblasts. ${ }^{9,10,29}$ Our finding that reticular and papillary dermal FRMs show different responses to SSR exposure further supports the notion that these FRMs may be synthesised by different cell populations. Similarly, it is feasible that fibroblasts and keratinocytes may synthesise and assemble FRMs differently, ${ }^{18}$ leading to differential binding of FRM-associated proteins. Recently, it has been identified that FRMs synthesised in vitro from human dermal fibroblasts lack MFAPs-2 and -4 and fibrillin-2 - key FRM-associated proteins. ${ }^{5}$

Fibrillin-rich microfibrils are particularly susceptible to UVR damage due to their photochemical composition; ${ }^{30}$ superficial microfibril assemblies in the papillary dermis ('oxytalan' fibres) are devoid of elastin and susceptible, by means of their amino acid content, to be particularly susceptible to UVR, ${ }^{19}$ whereas FRMs of the reticular dermis are largely protected, not only by their increased distance from the DEJ, but also by their combination with elastin (to form 'elaunin' fibres). ${ }^{6,31}$ Thus, differences in spatial arrangement, cellular origin, assembly and associated binding-proteins may drive FRM ultrastructural diversity and lead to differential responses upon exposure to SSR.

The ability of SSR to induce a more profound effect on papillary dermal FRM derived from African skin than those of Northern European skin was somewhat surprising. We hypothesise that these differences arise because FRM assemblies extracted from the papillary dermis of lighter pigmented subjects may have already accumulated damage in vivo. Papillary dermal FRMs appear particularly susceptible to photoexposure, due in part to their close proximity to the epidermis. ${ }^{10}$ Excessive sun exposure, mainly in childhood, and various high-risk activities, such as intentional sunbathing, inadequate sun protection and the use of tanning lamps by young individuals ${ }^{32,33}$ could all induce skin damage. It has been reported that almost all white Northern European individuals with normal recreational practices have sub-clinical signs of skin damage by the time they are 15 years old, ${ }^{34}$ which start to become discernible in their early 30 s. $^{35}$ In contrast, high levels of skin pigmentation are protective with regard to the cumulative effects of sun exposure; the melanin levels of basal keratinocytes afford a high protection factor ${ }^{15,16}$ resulting in little impact of UVR on keratinocytes or the 


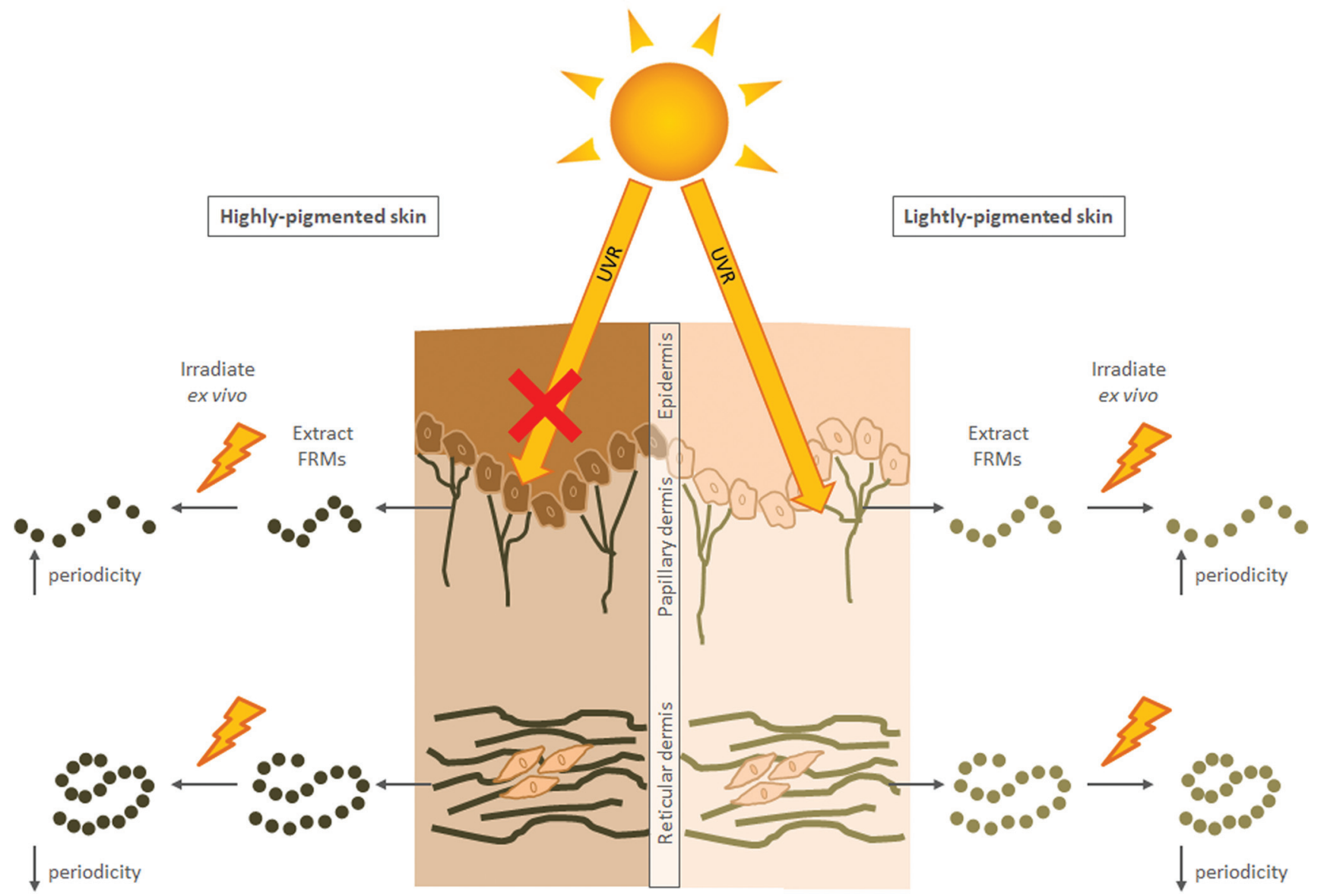

Fig. 3 Schematic image summarising our proposed hypothesis of the differential remodelling of FRM that occurs in response to SSR exposure. In this study we propose that incidental UVR exposure of photoprotected lightly-pigmented skin may induce sub-clinical damage to papillary dermal FRMs that induces remodelling of the FRMs. This damage may arise due to their close proximity to the DEJ and as a result of their low-level protection from melanin. Upon ex vivo SSR irradiation, these same FRMs are further remodelled and inter-bead periodicity significantly increases. In contrast, the papillary dermal FRM in highly-pigmented skin are well-protected by epidermal melanin and hence incidental UVR damage is potentially mitigated. As such, upon ex vivo SSR exposure these FRM are more susceptible to remodelling and inter-bead periodicity is increased to a greater magnitude. FRMs of the reticular dermis are largely protected from incidental UVR exposure in vivo due to their increased depth from the surface of the skin. Extraction of these FRM and subsequent SSR exposure causes a significant decrease to inter-bead periodicity as compared to unirradiated controls across both cohorts. We hypothesise that differences in spatial arrangement (papillary vs. reticular dermis), cellular origin (keratinocytes vs. fibroblasts), assembly and associated binding-proteins may drive FRM ultrastructural diversity and lead to their differential damage responses upon exposure to SSR.

dermal ECM. ${ }^{9,11,36}$ Hence, when extracted and subjected experimentally to SSR, papillary dermal FRMs undergo similar remodelling regardless of skin ethnicity, but with the caveat that those extracted from lightly-pigmented skin may have already been subject to remodelling at baseline (Fig. 3).

Elastic fibre biology is highly complex and such complexity presents a technical challenge in unravelling the multiple molecular interactions that FRMs possess. In this study, we demonstrate the direct effect of SSR exposure on FRM ultrastructure; an approach that provides a simplistic representation of the direct photochemical remodelling events that may occur in vivo. Although beyond the scope of the current manuscript, future studies should focus on understanding the consequences of UVR exposure on the molecular composition of
FRMs and how such remodelling may affect the role that FRMs play in skin homeostasis and cutaneous ageing.

\section{Abbreviations}

AFM atomic force microscopy

DEJ dermal-epidermal junction

ECM extracellular matrix

FRM fibrillin-rich microfibrils

FST Fitzpatrick skin type

MMP matrix metalloproteinase

ROS reactive oxygen species

SSR solar simulated radiation

UVR ultraviolet radiation 


\section{Funding sources}

This study was funded by a programme grant from Walgreens Boots Alliance, Nottingham, UK (awarded to CEMG, REBW and MJS). CEMG and REBW are supported in part by the National Institute for Health Research Manchester Biomedical Research Centre.

\section{Conflicts of interest}

The authors state no conflict of interest. Whilst supported in part by a programme grant from industry, funders had no editorial control over this manuscript.

\section{Acknowledgements}

We are grateful for the technical assistance of Dr Nigel Hodson from The University of Manchester BioAFM Facility. CEMG is a National Institute for Health Research (NIHR) Emeritus Senior Investigator.

\section{References}

1 S. H. Hussain, B. Limthongkul and T. R. Humphreys, The Biomechanical Properties of the Skin, Dermatol. Surg., 2013, 39, 193-203.

2 C. M. Kielty, M. J. Sherratt and C. A. Shuttleworth, Elastic fibres, J. Cell Sci., 2002, 115, 2817-2828.

3 R. P. Mecham and E. C. Davis, in Extracellular matrix assembly and structure, ed. P. D. Yurchenco, D. E. Birk and R. P. Mecham, Academic Press, New York, 1994, pp. 281-314.

4 D. R. Keene, B. K. Maddox, H. J. Kuo, L. Y. Sakai and R. W. Glanville, Extraction of extendable beaded structures and their identification as fibrillin-containing extracellular matrix microfibrils, J. Histochem. Cytochem., 1991, 39, 441449.

5 A. Eckersley, K. T. Mellody, S. Pilkington, C. E. M. Griffiths, R. E. B. Watson, R. O’Cualain, C. Baldock, D. Knight and M. J. Sherratt, Structural and compositional diversity of fibrillin microfibrils in human tissues, J. Biol. Chem., 2018, 293, 5117-5133.

6 G. Cotta-Pereira, F. Guerra Rodrigo and S. BittencourtSampaio, Oxytalan, elaunin, and elastic fibers in the human skin, J. Invest. Dermatol., 1976, 66, 143-148.

7 U. Afzal, R. E. B. Watson and A. K. Langton, Cover Image: Capturing the architectural beauty of the dermal elastic fibre network, Br. J. Dermatol., 2017, 177, 1141-1142.

8 A. K. Langton, M. J. Sherratt, W. I. Sellers, C. E. M. Griffiths and R. E. B. Watson, Geographical ancestry is a key determinant of epidermal morphology and dermal composition, Br. J. Dermatol., 2014, 171, 274-282.

9 A. K. Langton, M. Hann, P. Costello, P. Halai, S. Alessi, A. L. Chien, S. Kang, C. E. M. Griffiths, M. J. Sherratt and
R. E. B. Watson, Heterogeneity of fibrillin-rich microfibrils extracted from human skin of diverse ethnicity, J. Anat., 2020, DOI: 10.1111/joa.13217.

10 R. E. B. Watson, C. E. M. Griffiths, N. M. Craven, C. A. Shuttleworth and C. M. Kielty, Fibrillin-rich microfibrils are reduced in photoaged skin. Distribution at the dermal-epidermal junction, J. Invest. Dermatol., 1999, 112, 782-787.

11 A. K. Langton, H. K. Graham, J. C. McConnell, M. J. Sherratt, C. E. M. Griffiths and R. E. B. Watson, Organization of the dermal matrix impacts the biomechanical properties of skin, Br. J. Dermatol., 2017, 177, 818827.

12 M. F. Bennett, M. K. Robinson, E. D. Baron and K. D. Cooper, Skin immune systems and inflammation: Protector of the skin or promoter of aging?, J. Invest. Dermatol. Symp. Proc., 2008, 13, 15-19.

13 R. E. B. Watson, N. K. Gibbs, C. E. M. Griffiths and M. J. Sherratt, Damage to skin extracellular matrix induced by UV exposure, Antioxid. Redox Signaling, 2014, 21, 10631077.

14 G. J. Fisher, S. C. Datta, H. S. Talwar, Z. Q. Wang, J. Varani, S. Kang and J. J. Voorhees, Molecular basis of sun-induced premature skin ageing and retinoid antagonism, Nature, 1996, 379, 335-339.

15 D. Fajuyigbe, S. M. Lwin, B. L. Diffey, R. Baker, D. J. Tobin, R. P. E. Sarkany and A. R. Young, Melanin distribution in human epidermis affords localized protection against DNA photodamage and concurs with skin cancer incidence difference in extreme phototypes, FASEB J., 2018, 32(7), 3700-3706.

16 B. B. Shih, M. D. Farrar, M. S. Cooke, J. Osman, A. K. Langton, R. Kift, A. R. Webb, J. L. Berry, R. E. B. Watson, A. Vail, F. R. de Gruijl and L. E. Rhodes, Fractional Sunburn Threshold UVR Doses Generate Equivalent Vitamin D and DNA Damage in Skin Types I-VI but with Epidermal DNA Damage Gradient Correlated to Skin Darkness, J. Invest. Dermatol., 2018, 138, 22442252.

17 A. K. Langton, S. Alessi, M. Hann, A. L. Chien, S. Kang, C. E. M. Griffiths and R. E. B. Watson, Aging in Skin of Color: Disruption to Elastic Fiber Organization Is Detrimental to Skin's Biomechanical Function, J. Invest. Dermatol., 2019, 139, 779-788.

18 C. M. Kielty, M. J. Sherratt, A. Marson and C. Baldock, Fibrillin microfibrils, Adv. Protein. Chem., 2005, 70, 405436.

19 S. A. Hibbert, R. E. B. Watson, N. K. Gibbs, P. Costello, C. Baldock, A. S. Weiss, C. E. M. Griffiths and M. J. Sherratt, A potential role for endogenous proteins as sacrificial sunscreens and antioxidants in human tissues, Redox Biol., 2015, 5, 101-113.

20 M. J. Sherratt, C. P. Bayley, S. M. Reilly, N. K. Gibbs, C. E. M. Griffiths and R. E. B. Watson, Low-dose ultraviolet radiation selectively degrades chromophore-rich extracellular matrix components, J. Pathol., 2010, 222, 32-40. 
21 E. Hanssen, S. Franc and R. Garrone, Atomic force microscopy and modeling of natural elastic fibrillin polymers, Biol. Cell, 1998, 90, 223-228.

22 M. J. Sherratt, C. Baldock, J. L. Haston, D. F. Holmes, C. J. Jones, C. A. Shuttleworth, T. J. Wess and C. M. Kielty, Fibrillin microfibrils are stiff reinforcing fibres in compliant tissues, J. Mol. Biol., 2003, 332, 183-193.

23 M. Yaar and B. A. Gilchrest, Photoageing: mechanism, prevention and therapy, Br. J. Dermatol., 2007, 157, 874-887.

24 L. Rittie and G. J. Fisher, UV-light-induced signal cascades and skin aging, Ageing Res. Rev., 2002, 1, 705-720.

25 G. J. Fisher, H. C. Choi, Z. Bata-Csorgo, Y. Shao, S. Datta, Z. Q. Wang, S. Kang and J. J. Voorhees, Ultraviolet irradiation increases matrix metalloproteinase-8 protein in human skin in vivo, J. Invest. Dermatol., 2001, 117, 219-226.

26 C. S. Sander, H. Chang, S. Salzmann, C. S. Muller, S. Ekanayake-Mudiyanselage, P. Elsner and J. J. Thiele, Photoaging is associated with protein oxidation in human skin in vivo, J. Invest. Dermatol., 2002, 118, 618-625.

27 S. A. Hibbert, R. E. B. Watson, C. E. M. Griffiths, N. K. Gibbs and M. J. Sherratt, Selective proteolysis by matrix metalloproteinases of photo-oxidised dermal extracellular matrix proteins, Cell. Signalling, 2019, 54, 191-199.

28 J. L. Long and R. T. Tranquillo, Elastic fiber production in cardiovascular tissue-equivalents, Matrix Biol., 2003, 22, 339-350.

29 S. L. Haynes, C. A. Shuttleworth and C. M. Kielty, Keratinocytes express fibrillin and assemble microfibrils: implications for dermal matrix organization, Br. J. Dermatol., 1997, 137, 17-23.

30 S. A. Thurstan, N. K. Gibbs, A. K. Langton, C. E. M. Griffiths, R. E. B. Watson and M. J. Sherratt, Chemical consequences of cutaneous photoageing, Chem. Cent. J., 2012, 6(1), 34.

31 S. M. Mithieux and A. S. Weiss, Elastin, Adv. Protein. Chem., 2005, 70, 437-461.

32 M. C. Cercato, E. Nagore, V. Ramazzotti, I. Sperduti and C. Guillen, Improving sun-safe knowledge, attitude and behaviour in parents of primary school children: a pilot study, J. Cancer Educ., 2013, 28, 151-157.

33 R. Turrisi, J. Hillhouse, J. Robinson, J. Stapleton and M. Adams, Influence of parent and child characteristics on a parent-based intervention to reduce unsafe sun practices in children 9 to 12 years old, Arch. Dermatol., 2006, 142, 1009-1014.

34 B. G. Green and J. Bluth, Measuring the Chemosensory Irritability of Human Skin, J. Toxicol., Cutaneous Ocul. Toxicol., 1995, 14, 23-48.

35 W. F. Bergfeld, The aging skin, Int. J. Fertil. Women's Med., 1997, 42, 57-66.

36 M. K. Robinson, Population differences in skin structure and physiology and the susceptibility to irritant and allergic contact dermatitis: implications for skin safety testing and risk assessment, Contact Dermatitis, 1999, 41, 65-79. 